\title{
Construcción y fuentes utilizadas para los términos médicos en el Diccionario de Autoridades
}

\author{
Bertha M. Gutiérrez Rodilla \\ Universidad de Salamanca
}

INTRODUCCIÓN

Como es bien sabido, el primer diccionario académico, el llamado Diccionario de Autoridades, ha servido de modelo a la mayor parte de los diccionarios españoles durante más de dos siglos, desde que fuera publicado a mediados del XVIII. Existen algunos trabajos realizados sobre esta obra, no muy numerosos y de valor desigual ${ }^{1}$. El nuestro -que forma parte de un trabajo más amplio sobre los términos relacionados con la medicina en los diccionarios españoles del siglo XVIII 2 - pretende servir para aclarar algunos detalles referentes a la historia de ese diccionario y, de un modo concreto, a la de las fuentes utilizadas en su confección y a la del método seguido para realizarlo. Con los datos que presentamos en esta ocasión intentamos contribuir al tratamiento de esta área particular del léxico que es la de los términos médicos pero, además, creemos pueden servir para revisar algunas ideas que circulan sobre la manera de cómo se construye el primer diccionario académico.

LA CONSTRUCCIÓN DEL DICCIONARIO: EL MÉTODO Y LA PLANTA

Los fundadores de la Real Academia Española tuvieron que empezar afrontando el problema del método que debían emplear para confeccionar el diccionario. Dado que la manera de trabajar que se había empleado en otros países podía retrasar muchísimo la publicación de la obra -algo cuyo entusiasmo no se lo permitía- recurrieron a repartir-

1 Vid., por ejemplo, E. Cotarelo y Mori (1914), pp. 89-127; A. Pérez Goyena (1992), pp. 458481; S. Gili Gaya (1963); F. Lázaro Carreter (1980), pp. 83-148; F. Lázaro Carreter (1985); J. F. Val Alvaro (1992).

2 Algunos de los resultados de ese estudio se pueden encontrar en B. M. GutiérRez Rodilla (1993), pp. 463-512 o B. GutiérRez Rodilla (en prensa). 
se las letras o combinaciones de letras ( $A$ ante $b$, ante $c$, ante d, etc.) entre ellos -eran ocho los que se dieron a la tarea-. Cada uno debía hacer en primer lugar, la relación de las palabras que contenía la combinación que le había tocado, luego se encargaría de buscar testimonios escritos que las autorizasen y finalmente, había de enfrentarse con su definición. Estos aspectos individuales se completaban con una manera de constrastar el trabajo de todos ellos, mediante reuniones semanales -en principio los jueves, aun cuando hubo épocas en que tuvieron que reunirse un día adicional, el lunes- en las que discutían entre todos, corregían los errores y aprobaban el texto definitivo:

Para experimentar las plumas, se repartió parte de la letra $A$ en sus primeras combinaciones $A B, A C, A D$ las quales se sortearon entre los que se hallaron presentes, y cada uno se encargó de la que le tocó, para componerla segun su méthodo, y que se pudiesse elegir despues el que pareciesse mas conveniente para salir al público.[...] En el modo de trabajar el Diccionario (que yá se havía empezado entre todos) era dudoso el méthodo: [...] y el exemplo de haver tardado en Francia mas de sesenta años hasta empezar à imprimir, excluían semejante disposición. [...] Assi prosiguió la idea de encomendar à cada Académico una combinación de la $\mathrm{A}$, segun su siguiente letra, y se ordenó por entonces se hiciesse lo mismo en las tres que siguen $\mathrm{B}, \mathrm{C}, \mathrm{y} \mathrm{D}^{3}$.

Para que este método hubiese dado buen resultado tendrían que haberse cumplido las siguientes premisas: que todos ellos hubieran sido igualmente aptos para la tarea, que compartieran los mismos criterios y que trabajaran todos a la par ${ }^{4}$, lo que pertenece al género de las imposibilidades metafísicas. Lo más grave, sin embargo, fue que a pesar de los buenos propósitos y de las reuniones frecuentes, debió existir muy poca coordinación entre ellos en la realización de las diferentes partes que integran el diccionario. Esto se percibe en la inexistencia de una orientación común para abordar el contenido de las definiciones, que son de tantos tipos como lexicógrafos trabajaron en él. No hubo coordinación tampoco en la manera de citar a los autores y sus obras: para un mismo autor y una misma obra se nos remite en unas ocasiones a tal o cual folio, y en otras, a tal parte, capítulo, página, etc; puede, incluso darse el caso de que no se diga de dónde se ha tomado la cita. "Como cada obra se evacuó varias veces para sacar, sucesivamente, las palabras correspondientes a cada combinación, y como cada redactor utilizó la edición que tenía a mano, se produjo un irremediable pandemonium en las citas" 5 .

Las divergencias las encontramos también, en los autores utilizados por los diversos realizadores del diccionario: mientras que hay unas obras consultadas por todos -las que en el cómputo general obtienen mayor número de citas- como ocurre con las de Francisco de Quevedo, Luis de Granada, Miguel de Cervantes, Andrés Laguna, o Juan Fragoso, por ejemplo, la mayor parte de ellas queda al libre albedrío del encar-

3 R.A.E. (1726-1739-1963-), prólogo t. I, pp. XII y XIX.

4 F. Lázaro CARRETER (1980), p. 10.

5 F. LÁzARo CARRETER (1980), p. 111. 
gado de realizar cada letra. No podemos pasar por alto algunos hechos que son, al menos, llamativos: de Francisco de Quevedo y Villegas hay referencias frecuentes en todas las letras, excepto en la $\mathrm{F}$ y en la $\mathrm{M}$, en que sólo se le utiliza tres veces, para la primera y dos, para la segunda; el autor de la F, fue Vinzencio Squarzafigo, y el de la M, Diego Villegas y Quevedo. Joseph Casani, teóricamente encargado de la $\mathrm{CH}$, I, J o Y, no acude a Miguel de Cervantes, como hace el resto de lexicógrafos, pues no encontramos ejemplos suyos en los términos relacionados con la medicina pertenecientes a estas letras, salvo ijadear. Lo mismo ocurre con Carlos de la Reguera con respecto a Fray Luís de Granada, puesto que no hay ninguna voz de la medicina que avale este autor -excepto zumbido-, que empiece por $\mathrm{K}, \mathrm{R}, \mathrm{o} \mathrm{Z}$-letras que le habían correspondido a él-. El único autor de quien parece que no prescindió nadie, fue Andrés Laguna.

Se nota así mismo que los mismos lexicógrafos, al consultar determinadas obras para definir la parte de vocablos que le había tocado, podían hacer llegar algunos ejemplos particularmente interesantes, a quienes se enfrentaban con la definición de otras partes. Y hasta es posible comprobar a través de los resultados que en esta obra, como en todas las de gran magnitud y disparidad de colaboradores, siempre hay unos entusiasmados e infatigables que hacen su trabajo con prontitud; suele tocarles después a ellos echar una mano a los más premiosos... ${ }^{6}$

Al extraer de este diccionario los términos relacionados con la medicina y las fuentes que los ilustran, hemos encontrado datos como los siguientes: la Crónica general de España fue muy empleada por los autores de las letras A a la G, pero no aparece a partir de ahí más que en una ocasión, para el término molar. Con la finalización del tomo primero se deja de utilizar a Martín de Arredondo y su Recopilación de Albeytería sacada de varios autores...(1658), la Historia de Don Belianís ${ }^{7}$, la Suma de Fueros de Aragón de Bernardino Calvo, la obra de Juan Bautista Dávila Passion del Hombre-Dios referida y ponderada en Decimas Españolas (1661), el Servidor de Albuchasis traducida del árabe al latín por Simón Ginovés y del latín al castellano por Alonso Rodríguez

6 Los prólogos de los volúmenes primero y sexto, nos proporcionan la relación de los lexicógrafos que realizaron cada letra o cada combinación, a partir de la $C$ : Andrés González de Barcia, la combinación $C A$; Manuel de Villegas y Piñateli, la $C A, C O$ y $O$; Tomás Montes y Corral, $C E$ y $C H$; Joseph Casani, la $C H, D, Y, J, Y$ (tras la muerte de Juan de Villademoros y Fernando Bustillo); Lorenzo Folch de Cardona, $C E, C l$ y $D$ (tras la muerte de Juan de Villademoros y Fernado Bustillo); Fernando Bustillo y Azcona, $C O, D, H, L$ y $P$; Miguel de Perea, $C O$; Juan de Villamoros, D; Adrian Connink, la $E$ y $C L$; Vincencio Squarzafigo, la $F, C U, D$ (tras la muerte de Juan de Villademoros y Fernando Bustillo) y $P$ (tras la muerte de Fernando Bustillo); Juan de Ferreras, $G$; Manuel de Villegas Piñateli, $H$ y $O$; Pedro Serrano, $L$ y $R$; Joseph Torrero, $L$; Diego Villegas y Quevedo, $M$; Carlos de la Reguera, $D, N, K, R I$, $R O$, y $Z$; Jacinto de Mendoza, $P$ (tras la muerte de Fernando Bustillo); Francisco Antonio Zapata, $Q$; Pedro González, RU; Diego Suárez de Figueroa, $S$; Lope Hurtado de Mendoza, $T$; Miguel Gutiérrez de Valdivia, $U$ y $V$; Blas Antonio Nasarre, $X$. Esto es lo que figura en los prólogos, otra cosa es que se cumpliera. La información sobre este punto la proporciona F. Lázaro Carreter (1980) en el capítulo, ya citado, pp. 113-121.

7 Aunque así es como aparece recogida y no figura quién sea su autor, suponemos que se refiere al Libro del valeroso e invencible Príncipe Don Belianís de Grecia, de Jerónimo Fernández, impresa en 1547. 
de Tudela (1516), la Sevillana Medicina de Juan de Avignon, traducida del latín por Nicolás Monardes (1545) y la Doctrina moderna, para los Sangradores... de Ricardo Le Preux (1717). La Cirugía Sagrada de Francisco Suárez de Ribera (1726), sólo es manejada por los definidores de la letra $\mathrm{C}$, mientras que a Sorapán de Rieros lo utilizan exlusivamente para la $\mathrm{F}$, la $\mathrm{L}$ y la $\mathrm{S}$. La poesía de Fernando de Herrera inspira a quien atiende a la L y también, aunque menos, al de la E, M y T. A Benito Jerónimo Feijoo, sólo lo utiliza el encargado de la S, de la misma forma que las catorce referencias registradas de la Primera parte de Medicina y Cirugía racional y espagírica... de Juan de Vidós y Miro (1691), apoyan todas ellas, términos de la S. A la obra de Juan de Avila se hace referencia en una sola ocasión dentro de la letra $C$ y ya no vuelve a aparecer hasta la V, que es cuando aparece también la obra de Gregorio López. Debe quedar bien entendido que todo lo que decimos solamente tiene validez desde el estudio de los términos relacionados con la medicina, pero que puede no tenerla para la totalidad de los términos de la obra, o incluso para cualquier otro grupo de términos diferente al que nosotros hemos estudiado 8 .

Con las precauciones que he señalado, parece que se pueden tomar como ejemplos claros para entender las consecuencias del modo de trabajar de quienes hicieron el Diccionario de Autoridades, hechos como que el libro Bocados de oro aparezca en la lista de obras utilizadas de los tomos primero, tercero, cuarto, quinto y sexto, pero no del segundo, el que corresponde a la C, como la de B. Jerónimo Feijóo lo hace en las de los tomos segundo, tercero, cuarto y sexto, pero no en la del primero ni en la del quinto $^{9}$. Sorprende también que Juan Fragoso no aparezca en la lista de autores utilizados del tomo primero, sí en las de todos los demás, y sin embargo, aparezca ilustrando términos como alantoides, albarazo, almaro, alhurreca, algalia, almorrana o alhorra, pertenecientes todos ellos al tomo primero. Es aún más llamativo que sea en el prólogo del tomo cuarto cuando queda constancia de la autorización del empleo de la obra de Martín Martínez para ilustrar las voces del diccionario: ya se incluía en las listas de autores utilizados de los tomos segundo y tercero. Esto parece demostrar que una cosa eran las directrices aprobadas en las reuniones y otra muy distinta lo que cada uno fuera haciendo en su práctica lexicográfica.

Pero, incluso, esas directrices debieron darse de forma bastante precipitada en algunas ocasiones y experimentaron cambios a lo largo de la redacción de la obra, pues

8 No podemos cotejar con otros trabajos realizados pues no sabemos de la existencia de ningún estudio hecho en este sentido.

9 De hecho a este autor, y en lo que a términos relacionados con la medicina se refiere, sólo lo usó el encargado de la $S$, que es asimismo el único que utilizó la obra de Juan de Vidós, como ya hemos dicho. Este mismo autor parece que no gustaba de la obra de Fragoso, a quien sólo recurrió en tres ocasiones. Las 13 veces que recurrió a la Anatomia completa de Martín Martínez, no se preocupó de documentar las voces en el texto, sino que con apoyarlas en el índice le bastó, si bien fue el único lexicógrafo de todo el diccionario que utilizó la Medicina Scéptica de este mismo autor, para apoyar 19 términos en él y todos perfectamente localizados. Además de lo anterior, hay que decir del encargado de la $S$, Diego Suárez de Figueroa, que se tomó en serio su trabajo: sólo un 12’32\% de los términos relacionados con la medicina que comienzan por la letra $S$, se quedaron sin fuente que los apoyara. Esuno de los porcentajes más bajos de todas las letras. 
de otro modo no se entiende cómo se puede incluir en el tomo segundo, el de la C, a un autor como Manuel de Porras, casi coetáneo de los realizadores del diccionario, y desde ese momento, en los tomos tercero, cuarto, quinto y sexto se prescinda de él ${ }^{10}$. Ricardo Le Preux, que también vivió en esa época, autor de un libro sobre odontología, es utilizado en el primer tomo aunque a partir de ahí no se le vuelve a consultar. Si no carece de sentido evitar las obras, por ejemplo, de Juan de Avignon en el tomo segundo -para dar entrada a autores más modernos- a pesar de haber sido empleado en el primero, no es fácil entender que se cuente en los tomos tercero y cuarto con el Lilio de medicina... de Bernardo de Gordonio cuya traducción -sin autor conocido- desde el latín es de 1495 y con el que ni siquiera se había contado para los tomos primero y segundo. Siendo Bernardino Montaña de Montserrate el primer anatomista español que escribe su obra de anatomía totalmente en romance, no parece coherente con la idea académica de limpiar nuestra lengua y devolverla a su pureza, el que sólo se le utilice para el tomo segundo. La obra de Juan B. Juanini aparece en la lista de autores utilizados de los tomos primero, cuarto y quinto y no en la del segundo, tercero y sexto ${ }^{11}$; otro tanto pasó con la obra de Fernando Infante, autorizada en los tomos segundo y tercero, pero no en el primero, cuarto, quinto y sexto ${ }^{12}$.

Es razonable que en el tomo primero del diccionario se utilizara la Practica en cirurgia... de Juan de Vigo (¿1535?), y que a partir del segundo se usara la traducción hecha al castellano de esta misma obra por el Doctor Miguel Juan Pascual (1537), pero no se entiende, en cambio, que la Historia de la composición del cuerpo humano de Juan Valverde de Amusco (1556) no se tuviera en cuenta hasta el tomo cuarto ${ }^{13}$, puesto que, como es bien sabido, Valverde es uno de los creadores de nuestro léxico anatómico al proponerse introducir y acuñar científicamente términos extraídos de la lengua popular; es decir, pretende expresar de forma científica sus conceptos anatómicos, con palabras netamente romances. A lo largo de su obra se percibe esa tendencia vulgarizadora, a la vez que simplificadora, de la denominación anatómica ${ }^{14}$ : "Es literalmente pasmoso el audaz desenfado con que nuestro romancista convierte en nombres técnicos los vocablos más vulgares de la lengua vulgar y se enfrenta con las opiniones más acreditadas"15. A Manuel de Porras lo acusó el propio Martín Martínez de utilizar un léxico culto y afrancesado, hasta el punto de ser ésta la crítica principal que haga a su obra Martín Martínez ${ }^{16:}$ "El Dr. Porras creyó hacer un servicio á los cirujanos romancistas publicando esta obra; pero intercaló en ella cuestiones, impertinentes unas,

10 Si bien en la $S$, nos encontramos dos términos apoyados en M. de Porras. También puede ser que alguno de los que hizo la $C$, tomó nota de esos dos términos para pasársela al compañero al que le tocara hacer la $S$.

11 En la realidad debió ser poco utilizado, pues sólo lo hemos encontrado apoyando dos términos: babeo e incindente.

12 De hecho parece que sólo lo utilizaron en el tomo segundo.

13 Aunque no se tuvo en cuenta sólo "oficialmente" porque, de hecho, hay un término de la $A$, es decir, del primer tomo, documentado en Valverde de Amusco.

14 L. AlberTI LóPEZ (1948), p. 209.

15 P. Laín Entralgo (1946), p. 210.

16 L. SÁnChez GRANJel (1963), p. 41 
é inútiles otras, y se valió de ciertas voces oscuras y latinizadas, que sirvieron de motivo al sutil Martin Martinez para impugnarle en sus Noches anatómicas, que escribia ese mismo año". ${ }^{17}$. Este último doctor estaba radicalmente en contra de cultismos y galicismos -aunque era una oposición meramente teórica pues en sus propios escritos no fue tan exigente consigo mismo como pedía a los demás que lo fueran-. Su deseo era que se volvieran a emplear los términos utilizados en el Renacimiento y que se recuperaran las expresiones populares del español. "Es decir, frente a culteranismo y afrancesamiento se levantaba casticismo y purismo. Castizo y purista quiere ser Martínez resucitando la nomenclatura de Valverde, recomendando el uso de las llanas palabras del vulgo, para ganar así un puesto entre las autoridades de la lengua, al lado del anatómico de Amusco"18. Esto explicaría claramente la inclusión de Valverde y la exclusión de Porras, pero no que aquella se hiciera a partir del tomo cuarto y que ésta fuera desde el tercero; podría explicar incluso, la utilización de B. Montaña de Monserrate, por su casticismo, pero no que esta utilización se hiciera solamente en un tomo, en el segundo, que es precisamente el mismo en el que se incluye a Manuel de Porras, el "culto latiniparla" de Martín Martínez. Los criterios generales empleados no son, pues, fáciles de entender.

Además del método, y muy en relación con él, había que elaborar un proyecto que hubiera que seguir, una planta a la que atenerse. El encargado de realizar la primera de ellas fue Andrés González de Barcia que se encomendó afanosamente a la tarea y la tuvo acabada en muy poquito tiempo ${ }^{19}$. En esa primera planta se recogían normas sobre ortografía, distinciones sintácticas, las diferentes "marcas" que se debía aplicar a cada tipo de palabra o, por ejemplo, qué palabras debían incluirse y cuáles no. Muchas de estas normas se cumplieron a rajatabla pero muchas otras fueron continuamente contravenidas, sobre todo aquellas que se ocupaban de la inclusión o no de determinadas voces. Así por ejemplo, no se respetó el acuerdo de sustituir las palabras anticuadas por otras más modernas, con lo que el diccionario está lleno de arcaísmos que ya lo eran cuando fue realizado: abeñulas, alipte, barriguilla, cegal, cuer, physico o zurujano son algunos ejemplos de palabras en cuyas definiciones se explica que se trata de voces anticuadas o de poco uso. Son mucho más frecuentes en los dos primeros volúmenes, correspondientes a las letras A-C, que en el resto.

Tampoco se hizo mucho caso de la "orden" de desterrar las voces nuevas o inventadas en favor de la restitución de las antiguas, con más propiedad, hermosura, o mejor sonido. Esto permitió que entraran en el Diccionario de Autoridades términos como diartrosis o botiquín, así como muchas de las voces que tuvieron a bien inventar nuestros escritores, a la cabeza de los cuales figura Francisco de Quevedo.

Igualmente no sirvió de mucho que la primera norma que contiene la planta del diccionario excluyese "todas las palabras que significan desnudamente objeto inde-

17 A. HeRnández MoRejón (1842-1852), VI, p. 389.

18 C. DEL VAlle INCLÁN (1952), p. 194.

19 Esta planta se puede leer en el Diccionario de Autoridades, prólogo t. I, pp. XV-XVIII. Sobre la elaboración de la planta y los avatares que sufrió posteriormente véase, sobre todo, F. LAZARO CARRETER (1980), pp. 83-148. 
cente" -entendiendo por indecente, no sólo lo que lo es en su sentido más estricto, sino también lo deshonesto o lo sucio-, pues en este punto, como en tantos otros, el proyecto no se cumplió. Parece que les resultara difícil a los primeros académicos delimitar y separar lo que era indecente de lo que no lo era, aunque incluso cuando tenían clara esa separación, ello no les impedía introducir ese tipo de términos. Así, por ejemplo, en la palabra cuyo significado es "evacuar el vientre" se explica que:

porque esta voz y las que usa el vulgo, aunque proprias y expresivas, son indecentes y suenan mal, se substituyen las de obrar ò hacer del cuerpo

pero, a pesar de esto, la introdujeron.

Los propios lexicógrafos académicos fueron conscientes de que la planta estaba bien como punto de partida, pero que necesitaba una reforma rápidamente y así, tan sólo tres meses después de aprobada la primera quedan comisionados para presentar con urgencia una planta nueva, junto con Barcia -autor de la anterior-, Alcázar, Casani y Squarzafigo ${ }^{20}$. Pero también esas nuevas plantas o correcciones a la planta primitiva fueron igualmente violadas sin sonrojos. Por ejemplo, una vez publicados los dos primeros tomos del diccionario, Cardona denunció que se había procedido muy a la ligera en el punto referente a las palabras indecentes, y que había que intentar corregirse para los tomos que faltaban, de forma que propuso, y así se aprobó por votación, que no entrara ni una sola palabra más en el diccionario cuya simple mención pudiese molestar, o bien que no se pudiera definir sin "ofender los oídos de los más circunspectos":

Con los vocablos sucios se harán tres grupos; mantendrán su franquía las voces "comunísimas" y las que se limpian al entrar en ciertos sintagmas, como "culo de pepino"; pero la escoba será implacable para todas las malolientes invenciones de la "gente soez", algunas de las cuales se han colado ya, por indisculpable complacencia escatológica, en los tomos publicados ${ }^{21}$.

Esta decisión, sin embargo, no impidió en modo alguno que a partir del tomo segundo, es decir, de la $\mathrm{C}$ en adelante, siguieran incorporándose todos estos términos sin ningún pudor, muchos de ellos documentados en la obra de Francisco de Quevedo. Es decir, la propia experiencia fue la que marcó continuamente el camino a seguir y, por ella, se vieron obligados tantas veces a variar el rumbo. Es ésta, al parecer, una constante de cualquier trabajo lexicográfico.

20 F. Lázaro Carreter (1980), p. 107.

21 Así consta en las actas dela reunión de 22 de febrero de 1731, según F. Lázaro CARRETER (1980), p. 111. 
AUTORIDADES QUE ILUSTRAN LOS TÉRMINOS MÉDICOS 22

Fue aquella una ocasión en la que se trabajó en el dominio de la lexicografía de una manera que pocas veces se ha vuelto a repetir en España, por algo tan simple como era contar con un amplio fichero. Claro está que tal fichero, de acuerdo con las ideas de la Ilustración, estaba realizado tomando en su mayor parte ejemplos literarios ${ }^{23}$; aunque no todos los que ilustran los términos están sacados de la literatura, pues se recurre a otras ramas de las ciencias y las letras; aparte de que no todos los términos que se encuentran, están apoyados en una autoridad que los utilice: de las 2912 voces que aparecen en este diccionario relacionadas con el mundo de la medicina, 870, es decir, un $29,87 \%$, no están refrendadas por un autor que las avale. Este porcentaje no es el mismo en todas las letras, sino que varía de unas a otras, lo cual no deja de ser lógico puesto que, como ya se ha dicho, se encomendó la realización de cada parte a personas diferentes.

\section{Fuentes no médicas}

De las autoridades no vinculadas a la medicina que ilustran los términos médicos de que consta esta obra, destacan dos sobre todos los demás, por el número de veces que se recurre a ellos. Se trata de Francisco de Quevedo (1580-1645), el más citado de todos los autores literarios ${ }^{24}$, al que se acude en 162 ocasiones, y Fray Luis de Granada (1504-1588), que le sigue en cuanto a apariciones, 121. Tras ellos, está Miguel de Cervantes (1547-1616), documentando 96 términos. Las obras utilizadas de estos escritores son prácticamente todas las que escribieron, aunque también en esto hubo sus preferencias: los poemas de Quevedo se citan mucho más que el resto de su obra en prosa; entre los libros de Luis de Granada el Símbolo de la fe es el preferido y el Quijote, entre los de Cervantes.

Que sea Quevedo el autor no médico más utilizado no hace sino confirmar que no existió una actitud antibarroca en los primeros académicos, por más que la Academia Española hubiera surgido para

defender la lengua, exclusivamente, de los barbarismos, léxicos y sintácticos, introducidos por las agonizantes escuelas barrocas 25

22 Los datos que ofrecemos ahora han sufrido algunos cambios respecto a los que ya presentamos en B. GutiérRez Rodilla (en prensa), pp. 1.027-1.038. Las diferencias se deben a una segunda revisión realizada sobre el diccionario.

23 Era lógico que así fuera pues el fin que se perseguía era, ni más ni menos, que dotar al español de una norma.

24 No es extraño que sea Quevedo el autor no médico más citado puesto que en su obra afloran muy a menudo los asuntos médicos, normalmente para ser sometidos a su aguda crítica.

25 Acta de la reunión mantenida el 3 de agosto de 1713 por los primeros académicos. (Cito por F. LÁzaro CARRETER (1985), p. 214). 
Como bien explica Lázaro Carreter, no se puede identificar el origen del movimiento academicista con el purismo lingüístico, o el casticismo literario posteriores. La Academia no se opone a un gusto literario, cuyos logros son motivo de orgullo, sino que va a tratar de cortar la barbarie que la degeneración de esos gustos ha causado. "Porque no había surgido el movimiento clasicista, que hace de todo el XVII el siglo de la barbarie, y que erige en Siglo de Oro al XVI, la Academia puede poner en las listas de las autoridades del idioma los nombres, después repudiados, de Quevedo, Paravicino, Pellicer, Góngora, Ulloa, Carrillo o Calderón. Estos escritores no han llevado la lengua a un caos, y su voz debe ser escuchada, porque es magistral, en la misma medida que la de Garcilaso o fray Luis"26. En cualquier caso, frente a las 162 citas de Quevedo, sólo hay 31 de las obras de Góngora.

Podría sorprender, en principio, que sea Luis de Granada el segundo autor no médico más utilizado, pero ya ha sido señalado por Laín Entralgo que precisamente es él uno de los creadores de nuestro léxico anatómico castizo, junto con Montaña de Monserrate y Valverde. Es el de Amusco, en opinión de Laín, quien sirve de guía al dominico aunque éste no cae, no obstante, en los extremos del osado "anatomista castellano": los términos empleados por fray Luis son fieles al romance, pero "su buen gusto literario y su formación a pechos de los clásicos y de escritores en latín le moverían, sin embargo, a dulcificar o culteranizar las expresiones de Valverde que malsuenan a sus oídos"27.

Mucho más distanciadas, en cuanto a la frecuencia de aparición, son las obras de otros escritores como F. Lope de Vega (1562-1635), al que se acude en 79 ocasiones, 59 como tal Lope de Vega y 20 con su seudónimo "Tomé de Burguillos"; Pedro de Ribadeneyra (1527-1611) (47); la traducción hecha por Diego Gracián en 1548 de las Morales de Plutarco (45); J. Eusebio Nieremberg (1595-1658) (44); Luis de Góngora (1561-1627) (31); Anastasio Pantaleón (1600-1629) (29); Antonio de Solís (1610-1686) (29); Fray Hortensio F. Paravicino (1580-1633) (29); Vicente Espinel (1550-1624) (28); La vida y hechos de Estebanillo González. [...] Compuesta por él mismo (1646) (27); Salvador J. Polo de Medina (1603-1676) (27); José Pellicer (1602-1679) (25); Juan de Mena (1411-1456) (24); Mateo Alemán (1547-1614) (23); Francisco López de Ubeda (s. XVI-XVII) (23) ${ }^{28}$; Fray Antonio de Guevara (1480-1545) (22) o A. de Salas Barbadillo (1581-1635) (22), por ejemplo29.

Hay otro tipo de textos, no exactamente literarios sino más bien de tipo legal, de donde también se extraen ejemplos para los términos médicos de este diccionario: así $L a$ Pragmática de Tassas de 1680 a la que se acude en repetidas ocasiones -en 57 para las voces relacionadas con la medicina-, o La Nueva Recopilación de las Leyes del Reino, de donde salen ejemplos que acompañan a 27 de ellos; pertenecen a este mismo apartado,

26 Vid. F. LAzaro CarReter (1985), p. 215.

27 P. Laín Entralgo (1946), p. 212.

28 La presencia aquí de estos dos autores parece lógica puesto que ambos -M. Alemán y F. López de Ubeda- eran médicos. En este sentido, vid. L. CoRTEJoso (1969), pp. 3-23.

29 Sólo recojo en este apartado de fuentes literarias, los nombres de aquellos que tienen más de 20 referencias, pues, de otra forma, la lista sería interminable. 
pero son muchísimo menos frecuentes, la Crónica general de España (18), el Fuero Juzgo (8), Las siete partidas (6), Las partidas del Rey don Alonso (4), la Crónica de San Fernando (3), el Fuero real de España (2), la Crónica de los Reyes Católicos (1), la Recopilación de las leyes de Indias (1), el Ordenamiento real (1), las Pragmáticas reales (1), la Suma de fueros de Aragón (1), o las Ordenaciones de Tarazona (1).

Junto a estos se utilizan a menudo los datos del Tesoro de la lengua castellana (1611), de Sebastián de Covarrubias (125) y del Vocabulario latino-hispano (1492) y el Vocabulario hispano-latino (1495), de E. A. de Nebrija (58); también, con menor frecuencia, los que proporcionan el Arte para ligeramente saber la lengua araviga. Vocabulista aravigo en letra castellana (1505) de Fray Pedro de Alcalá (16), los Romances de germania de varios autores con su Bocabulario...(1609) de Juan Hidalgo (8), el Dictionarium, quanta maxima... (1531) de Ambrosio Calepino (2), o el Compendio para saber escogidamente latín con el uso elegante de... (1669) de Valeriano Requejo (1).

Tampoco son literarios, sino más bien de tipo histórico o político ${ }^{30}$, otro tipo de textos consultados para obtener referencias en que apoyar los términos. Entre ellos: la Historica relacion del reyno de Chile... (1646) de Alonso Ortiz de Ovalle, a quien se recurre en 42 ocasiones; la Historia natural y moral de las Indias (1590) del Padre José de Acosta (26); la Descripcion general de Africa (1573-1599) y la Historia de la rebelion y castigo de los moriscos del reyno de Granada (1600), de Luis del Marmol Carvajal (22); la obra completa de Ambrosio de Morales ${ }^{31}$, con 21 referencias; la Idea de un príncipe político cristiano representada en cien y una empresas (1640), la Corona gothica, castellana y austriaca... (1646) y la República literaria... (1655), de Diego Saavedra (17); Juan de Mariana y su Historia General de España, traducida por él mismo de su obra latina en 1601 (15); el Libro llamado silva de varia lección dirigido a la S. C. C. M. del Emperador... (1540), de Pedro Mexía (13); la Conquista de las Islas Molucas (1609), de Bartolomé Leonardo de Argensola ${ }^{32}$ (13); Alonso Martínez de Espinar y su Arte de Ballestería y monteria... (1644) (11); la Historia de la insigne Ciudad de Segovia, y Compendio de las Historias de Castilla (1637), de Diego de Colmenares (8); Fernando del Castillo y su Primera parte de la Historia general de Sancto Domingo, y de su orden de predicadores (1584), (8); la Guerra de Granada... (1627), de Diego Hurtado de Mendoza (8); Pedro López de Ayala y su Cronica del Rey don Pedro... (1495) (7); la Naturaleza, policía sagrada, profana, costumbres i ritos... (1627), de Alfonso de Sandoval (6); Francisco López de Gómara y su Historia General de Las Indias con todo el descubrimiento..., con la conquista de México y de la nueva España (1552) (6); o la Historia general de los hechos de los Castellanos en las Islas... (1601-1615) así

30 Somos conscientes de que estos apartados que establecemos dentro de "Fuentes no médicas" son un poco aleatorios puesto que muchos de estos escritores publican a la vez obras literarias e históricas, religiosas o políticas, por lo que, generalmente, resulta muy difícil encuadrarlos dentro de un solo grupo.

31 Se cita como obra completa pero no hemos encontrado ninguna edición de la obra completa de este autor que pudiera ser consultada como tal por los autores del diccionario. La primera de la que tenemos noticia es la recopilación y ordenación realizada por F. Valerio Cifuentes con el nombre de Opúsculos castellanos..., en fecha muy posterior -1793- a la realización del diccionario.

32 También se utilizaron otras obras suyas y de Lupercio L. de Argensola, de tipo literario. 
como la Primera parte de la Historia general del Mundo, de XVI años del tiempo del Señor Rey don Felipe II... (1601), de Antonio de Herrera Tordesillas (5), por ejemplo.

Otro grupo no menos importante, estaría constituído por obras fundamentalmente de tipo religioso o filosófico, tales como la Chrónica Seraphica. Vida del Gran Padre San Francisco... (1682-1698), de Fray Damián Cornejo (19); la Chronohistoria de la Compañia de Jesús... (1710) y la Vida, virtudes y milagros de San Julián, segundo Obispo de Cuenca (1692), de Bartolomé Alcazar (17); María Jesús de Agreda, y su Mystica ciudad de Dios... (1688) (15); la Primera parte de la vida de Christo Señor Nuestro (1596) y el Tratado del amor de Dios (1592), de Fray Cristobal Fonseca (14); Martínez de la Parra y su Luz de verdades católicas... (1691) (13); la Vida de San Gerónimo... (1595) y la Historia de la Orden de San Gerónimo... (1600-1605), de José de Sigüenza (12); Martín Navarro de Azpilcueta y su Manual de confesores... (1549) (11); la Vida y hechos de Pio V... (1595), de Antonio de Fuenmayor (11); Luis Muñoz y sus obras Vida de Bartolomé de los martires... (1614), Vida de $S$. Carlos Borromeo... (1626), Vida y virtudes del Venerable Fray Luis de Granada... (1639), Vida De la Venerable M. Mariana de S. Joseph... (1645) y la Vida y virtudes del V. P. Camilo de Lelis (1653) (9); las Obras... (1659-1671), de Juan Palafox y Mendoza (9); la Philosophia moral de principes... (1596), de Juan de Torres (9); Fernando de Valverde y su Vida de lesu Cristo... (1657) (9); la Idea de el Buen Pastor, [..] representada en Empresas sacras... (1682), de Francisco Nuñez de Cepeda (7); el Governador christiano... (1612), de Juan Márquez (7); La heroyca vida, virtudes y milagros del grande San Francisco de Borja... (1702), del cardenal Alvaro de Cienfuegos (7); los Exercicios de perfección... (1609), de Alonso Rodríguez (7); la Primera parte de las Postrimerías del hombre... (1603), de Pedro de Oña (6); la Historia Pontificial y catholica... (1565), de Gonzalo de Illescas (5); Alejo de Vanegas y su Agonía del transito de la muerte... (1537) (5), o la Recopilacion de todas las obras... (1554) y el Epistolario christiano... (1567), de Alonso de Orozco (5), entre otras.

Por último, un pequeño apartado constituído por libros de botánica, matemáticas, divulgación científica, etc. tales como la Obra de agricultura copilada de diversos auctores... (1513), de Gabriel Alonso de Herrera (16); la traducción -publicada en 1621que de la Historia de aves y animales de Aristóteles, hizo Diego de Funes (5); la Plaza universal de todas las ciencias (1615), de Cristóbal Suárez de Figueroa (5); el Compendio Mathematico (1707-1715) y el Compendium philosophicum (1721), de Tomás Vicente Tosca (5); el Vocabulario del Humanista [...] de aves, peces, quadrupedos... (1569), de Lorenzo Palmireno (1), o los Quatro Libros de la Naturaleza y virtudes de las plantas, y animales que están recevidos en uso de medicina en la Nueva España (1615), del médico Francisco Hernández, (1), por ejemplo.

\section{Fuentes médicas}

Entre las fuentes médicas destaca por delante de todos los demás, Andrés Laguna y su traducción castellana con comentarios del Pedacio Dioscorides Anazarbeo, acerca de la materia medicinal, y de los venenos mortiferos... (1555), de forma que una sex- 
ta parte de las referencias a autores tanto literarios como no literarios para voces relacionadas con la medicina, se refieren a Laguna y a esta obra, 471 , que suponen además, el $51,30 \%$ del țotal de las ilustradas por autores médicos ${ }^{33}$, que son 918 . No hay constancia de que se utilizara ninguna otra de las obras escritas por A. Laguna. Los otros autores médicos a que se recurre por orden de frecuencia, son: Juan Fragoso, el segundo más citado después de Laguna (176) y del que se recogen obras como el Aromatum, fructuum, et simplicium aliquot medicamentorum... (1601), la Chirugia universal, emendada y añadida (1606) o De Succedaneis Medicamentis liber... (1632); Martín Martínez, que suma en total 125 referencias y sus obras Noches anatómicas, 0 Anatomía compendiosa (1716), Medicina scéptica y Cirugia moderna... (1722-1725), Examen nuevo de Cirugía moderna (1722), el Discurso físico sobre si las víboras deben regentarse... (1723) y la Anatomía completa del hombre (1728); Jerónimo Gómez de Huerta y la Traducción de los libros de Caio Plinio Segundo, de la Historia Natural... (1599), (46); la Historia de la composición del cuerpo humano (1556), de Juan Valverde de Amusco (35); la Primera parte de Medicina y Cirugía racional y espagírica... (1691), de Juan de Vidós y Miro (15); la Médula de Cirugía y examen de cirujanos (1691) y la Anatomía Galenico-Moderna (1716), de Manuel de Porras (9); la Medicina española contenida en proverbios vulgares de nuestra lengua... (1616), de Juan Sorapán de Rieros (8); la Nueva philosophia de la Naturaleza del hombre... (1587), de Miguel Sabuco ${ }^{34}$ (6); los Problemas... que tractan de cuerpos naturales y morales (1543) y el Dialogo del calor natural (1543), de Francisco López de Villalobos (6); la Cirugía Sagrada...(1726), de Francisco Suárez de Ribera (3); el Libro, o práctica en Cirurgía... de Juan de Vigo (3), en dos casos se especifica que se refieren a la traducción hecha de esta obra por el doctor Miguel Juan Pascual ${ }^{35}$ publicada en Valencia en 1537; el Libro de la anathomia del hombre... (1551), de Bernardino Montaña de Montserrate (2); la Magna y canonica cirugia de Guido de Cauliaco... traducida en 1658, por Fernando Infante de Avrioles (2); el Tesoro de medicinas, para todas enfermedades... (1672), de Gregorio López (2); las Obras Medicas de Juan Bautista Juanini $^{36}$ (2); el Lilio de Medicina de Bernardo de Gordonio ${ }^{37}$ (2); las Noticias de el caphe... (1692), de Juan de Tariol (1); Juan de Avignon y su Medicina Sevillana ${ }^{38}$ (1); la

33 El porcentaje se desvía tanto hacia Andrés Laguna porque además de utilizarlo para ilustrar otros términos relacionados con la medicina, se incluyen en el diccionario muchísimas de las plantas -con uso medicinal o no- que aparecen en su obra. De entre ellas sólo hemos contabilizado aquellas que tienen aplicación terapéutica.

34 Ciertamente no se trata de un libro de medicina pero sí es una obra antropológica escrita por un médico; sobre esto vid. L. SÁnCHEz GRANJel (1956).

35 No utilizaron, sin embargo, su Morborum internorum, que es uno de los mejores tratados de medicina práctica publicados en la España del XVI.

36 No encontramos en ningún repertorio una edición de los escritos de J. B. Juanini bajo el título de Obras Medicas.

37 Seguramente se utilizaría la traducción hecha desde el latín en 1495.

38 Siendo para la realización de un diccionario romance parece poco probable que se utilizara la obra original latina sino más lógicamente, la traducción hecha del latín al castellano por Nicolás Monardes, publicada en 1545. 
Doctrina moderna, para los Sangradores, en la qual se trata... (1717), de Ricardo Le Preux (1); el diccionario médico de Casteti ${ }^{39}$ (1). También se utilizó el Armamentarium Chirurgicum... (1653), de J. Sculteto (1), aunque no para apoyar el uso de una determinada palabra, sino para ayudarse a la hora de formular su definición. En algunas ocasiones (12), se recurre a los escritos del polemista farmacéutico, examinador del real protomedicato, Félix Palacios y Bayá, Palestra pharmaceutica chymico-galenica (1706), La Pharmacopea, triunfante de las calumnias... (1713) y la traducción que hizo en 1703, del Curso chymico del Dr. Nicolas Lemery...

Hemos de terminar nuestro recorrido por esas primeras páginas de la lexicografía moderna española. No nos sorprenden las contradicciones que se perciben en ellas ni la falta de coordinación entre los académicos, o la desigual atención que se concede a los distintos textos; ni debería extrañarnos, porque no hemos de perder la perspectiva histórica, que no sea el uso el que determine la elección del léxico médico que ha de contener el diccionario.

Algunas de éstas son pequeñas sombras, entre muchas luces: las que reflejan el interés por las palabras, la preocupación por la realidad que designan y el decidido empeño, sin escatimar ningún esfuerzo, por realizar una empresa irrepetible en el campo de la lexicografía española: el Diccionario de Autoridades.

\section{BIBILIOGRAFÍA}

AlBeRTI LÓPEZ, L. (1948): "La anatomía y lengua romance. La Nomenclatura Anatómica”, en La Anatomía y los anatomistas españoles del Renacimiento, Madrid, CSIC, pp. 193-214.

CoRTEJoso, L. (1969): "La aportación de los médicos escritores a la literatura española del siglo de oro", Medicina e Historia, 61, pp. 3-23.

CotARELo y MoRI, E. (1914): "La fundación de la Real Academia Española, y su primer director: don Juan Manuel Fernández Pacheco, marqués de Villena", BRAE, 1, pp. 4-38, 89-127.

GILI GAYA, S. (1963): La lexicografía académica del siglo XVIII, Oviedo, Universidad de Oviedo.

GutiéRREZ RodiLla, B. M. (1993): "Los términos relacionados con la medicina en el Diccionario de Autoridades", BRAE, 73/260, pp. 463-512.

GutiérRez Rodilla, B. (en prensa): "El léxico de la medicina en el diccionario de E. de Terreros y Pando", en Actas del III Congreso Internacional de Historia de la Lengua Española.

HeRnández MoRejón, A. (1842-1852): Historia bibliográfica de la Medicina Española, Madrid, Imp. viuda de Jordán e hijos, 7 vols.

39 Aunque así es como se cita en el Diccionario de Autoridades en el término rhachitis, no hemos podido verificar la existencia de tal diccionario y de tal autor. Creemos que se refiere al Lexicon medicum Graeco-Latinum ex Hippocrate, et Galeno desumptum, de Bartolomeo Castelli, publicado en 1598 y del que se hicieron muchas reimpresiones y reediciones a lo largo del XVII y XVIII. 
LAín EnTRAlGo, P. (1946): "Onomatología anatómica", en La antropología en la obra de Fray Luís de Granada, Madrid, CSIC, pp. 198-216.

LÁzARo CARRETeR, F. (1980): "El primer diccionario de la Academia", en Estudios de lingüística, Barcelona, Crítica, pp. 83-148.

LÁzaro Carreter, F. (1985): Las ideas lingüísticas en España durante el siglo XVIII, Barcelona, Crítica.

Pérez Goyena, A. (1922): "Contribución de los jesuítas al Diccionario de Autoridades (17261739)", Razón y fé, 63, pp. 458-481.

R.A.E. (1726-1739 -1963-): Diccionario de Autoridades, Madrid, F. del Hierro (ed. facsímile, Madrid, Gredos).

SÁnchez Granjel, L. (1956): La doctrina antropológica de Miguel Sabuco, Salamanca, Universidad de Salamanca.

Sánchez Granjel, L. (1963): Anatomía española de la llustración, Salamanca, Universidad de Salamanca.

Val Álvaro, J. F. (1992): Ideas gramaticales en el "Diccionario de Autoridades", Madrid, Arco/Libros.

Valle InClán, C. Del: "El léxico anatómico de Porras y de Martín Martínez", Asclepio, 4, pp. 141-228. 